\title{
LA INTERDISCIPLINARIEDAD COMUNICATIVA: UNA VÍA PARA EL DESARROLLO DE LA LENGUA MATERNA Y LA PROFESIONALIDAD DE LOS UNIVERSITARIOS EN CUBA
}

PhD. María Rodríguez Cosme

Universidad de Oriente

mrodriguezc@uo.edu.cu

\author{
MSc. Silvia Smith Salazar \\ Universidad de Oriente \\ ssmiths@uo.edu.ec
}

\begin{abstract}
Palabras claves: interdisciplinariedad, comunicación, Recibido: 31 de enero de 2017 competencia, aprendizaje.
\end{abstract}

Keywords: interdisciplinary, communication, competence, learning.
Aceptado: 01 de marzo de 2017

\section{RESUMEN}

Los problemas de la lengua tanto en el código oral como escrito, constituyen una irregularidad en los estudiantes de los centros educacionales e incluso en la educación superior, los cuales deben desarrollar la comunicación tanto oral como escrita de modo que demuestren competencia comunicativa, esencial para un mayor nivel de aprendizaje en las diferentes materias que reciben durante sus años en la universidad. Desde esta óptica el trabajo metodológico de los Departamentos y de los Colectivos de Disciplinas resulta esencial, para precisar en los aspectos teórico- didácticos del español que se revierta en las diferentes asignaturas, por ser el idioma el macroeje del currículo, y no deja de haber comunicación en todo el proceso de enseñanza aprendizaje. Por consiguiente, el objetivo del presente artículo es desarrollar una orientación teórico- didáctica desde el currículo en la carrera de modo que se promuevan modos de actuación en los estudiantes en formación para su futura labor profesional y que potencie la competencia comunicativa.

\section{ABSTRACT}

The use of the written and the oral expression, is a real problem for students, mainly in those who register in careers of higher education. From this perspective, the methodological work should be guided to theoretic and didactic aspects of the Spanish language in the different subjects that appear in the syllabus taking into account that the Spanish is the central language of the curriculum and is present during the teaching-learning process. Accordingly, the objective of the present article is to develop a methodological guide from an interdisciplinary view in the school, and in the course-year to improve the communicative skill in the students. 


\section{INTRODUCCIÓN}

Los problemas de la comunicación oral y escrita en los estudiantes de las universidades aún es un problema a resolver por todos los docentes, en aras de lograr el desarrollo de la lengua oral y escrita como habilidades macro de gran significación como profesionales. Por eso es importante, que los estudiantes en formación reciban un sistemático tratamiento en la carrera, que les posibilite modos de actuación acorde con el reto que deben lograr después de graduado.

Las habilidades escuchar, hablar, leer y escribir son habilidades comunicativas esenciales para el desarrollo de la lengua materna, las cuales cobran particular importancia desde la preparación del profesional en formación en las carreras, años y grupos, para su mejor desempeño profesional en el contexto laboral luego, y no basta con una estrategia para la lengua, esta debe irse particularizando a partir del diagnóstico en cada grupo y en correspondencia con el trabajo que sea capaz de realizar el colectivo pedagógico.

Por tanto, ofrecer una atención a través de: acciones, indicadores, folletos desde el componente curricular y laboral investigativo a nivel de carrera, es hoy una necesidad insoslayable de los profesores de la lengua en cada una de las carreras. Tradicionalmente la prioridad a la lengua materna ha estado centrada en los docentes del área humanística. Sin embargo, su buen uso debe ser una preocupación de todos los docentes, pues gracias a su desarrollo se producen niveles superiores de cognición y comunicación, y solo así se potencia el aprendizaje.

Por consiguiente, desarrollar una orientación teórico- didáctica de enfoque interdisciplinario en la carrera y en el año que estimule el desarrollo de la competencia comunicativa en los estudiantes de carreras universitarias es esencial para su futura profesión.

La política educacional cubana, ha prestado primordial atención a la lengua materna en todas las educaciones. Desde el año 1996 se proclamó como programa director, en este sentido, se explicaba a todos los docentes la necesidad de su tratamiento en cualquier disciplina en el proceso educativo.

Esta temática ha sido estudiada desde diferentes ángulos en aras de encontrar alternativas que propicien vías, en el plano teórico como metodológico, de modo que se ofrezcan modelos para un trabajo más exitoso con la comunicación entre profesor alumno- alumno- alumno, alumno- profesor.

Las razones pedagógicas antes expuestas, establecen además que el profesor dentro de sus funciones tiene la de ser un coordinador de la actividad docente, para ello, propicia el aprendizaje efectivo del grupo o los grupos, así como el cumplimiento de los métodos y las técnicas más convenientes para el desarrollo de la actividad.

Otras de sus funciones es de facilitador, vista como la función de promover, incentivar la participación del resto del grupo, ya sea de los otros profesores o de los alumnos, de modo que se pueda dirigir el proceso con un carácter dialógico, donde se respeten las opiniones y se aprenda a escuchar y respetar criterios. Por ello, sugiere técnicas o procedimientos que promuevan el análisis como proceso lógico del pensamiento, junto a las demás habilidades. 
Cada uno de los elementos que favorecen la comunicación ha sido abordado como herramientas por la asignatura Práctica Integral de la lengua española en el primer año, así como Estudios literarios en los últimos años, lo que potencia el mejor desarrollo de la competencia comunicativa en los estudiantes en formación.

Pero sería oportuno interrogarnos acerca de ¿qué sucede con los modos de actuación de los profesionales recién graduados en los contextos laborales? Se considera una regularidad la atención tradicional a la lengua haciendo énfasis solo en las palabras técnicas de cada asignatura, cómo se escriben, qué significan, sin percibir que el discurso, los tipos de textos, la coherencia y cohesión como aspectos esenciales del texto entre otros aspectos medulares, no son tenidos en cuenta a la hora de evaluar a los estudiantes universitarios, en sus actividades docentes y en todos los contextos comunicativos.

\section{DESARROLLO}

\section{Algunas consideraciones acerca de la interdisciplinariedad y su enfoque comunicativo en Cuba}

Desde esta óptica resultó vital a través del trabajo metodológico realizar actividades que ofrezcan las vías para una mejor atención teórico-didáctica por todos los docentes, sin que se obviara brindar primero los contenidos teóricos abordados en la didáctica de la lengua y el cómo que es lo que aún falta en el modelo del profesional y en las diferentes disciplinas incluyendo la asignatura Práctica Integral de la lengua española.

Por tanto se partió de la realización de un taller metodológico que permitiera un acercamiento a los fundamentos acerca de la interdisciplinariedad. Al respecto se retomaron los estudios realizados en el país, por Cayetano Caballero Camejo quien valora que "El estudio del término disciplina permite indagar en la concepción acerca de la interdisciplinariedad" ( Caballero; 2004, p.4 )y, para ello, es necesario comenzar retrospectivamente, pues ya desde los tiempos del pedagogo humanista y filósofo checo Jean Amos Comenius, aparecen las ideas acerca de la articulación entre las asignaturas o entre conocimientos, y él plantea que se hace necesaria la relación entre las asignaturas, para poder reflejar un cuadro íntegro de la naturaleza en los alumnos, y además crear un sistema verdadero de conocimientos y una correcta concepción del mundo, desde esta óptica.

Cayetano valora el criterio de este gran pedagogo y afirma "que se enseñan muy mal las disciplinas cuando su enseñanza no va precedida de un vago y general diseño de toda la cultura, pues no hay nadie que pueda ser perfectamente instruido en una ciencia en particular sin relación con las demás" (Caballero, 2004, p.5) se refería ya a la relación necesaria que se produce entre las materias.

Jean Piaget en 1970 alude a interdisciplinariedad como la búsqueda de estructuras más profundas entre los fenómenos y que esté diseñada para explicar estos" (Barrera, 2007, p.24) obsérvese la carga psicológica de esta definición, al abordar la búsqueda del razonamiento a través de los procesos lógicos del pensamiento, todo lo que redunda en el desarrollo del conocimiento. 
La UNESCO en 1987 confirma que interdisciplinariedad es "el encuentro y la cooperación entre dos o más disciplinas, cada una de ellas contribuyendo a nivel teórico y empírico con sus esquemas conceptuales propios, su manera de definir los problemas y sus métodos de análisis" (Fiallo, 1999, p. 26). Jorge Fiallo sistematizando estos estudios plantea "es un proceso y una filosofía de trabajo, es una forma de pensar y de proceder para conocer la complejidad de la realidad objetiva y resolver cualquiera de los complejos fenómenos que esta plantea”. (Fiallo, 1999, p. 26)

En ambas definiciones se declara que es una necesidad insoslayable para resolver los fenómenos que se plantean a partir de métodos concretos. Estos análisis corroboran que en el proceso docente educativo se revelan situaciones de aprendizaje que parten de la realidad natural y social y que debe resolverse a través de la interdisciplinariedad.

González (1995) considera, que la función principal de la escuela es la comunicación; así como el aprendizaje es un tipo de comunicación donde la primera y más importante de las dificultades es la referida al lenguaje. Desde esta mirada se asume un criterio defendido por Barrera quien destaca "Las realidades nuevas no pueden ser designadas o nombradas con términos viejos, pues, al hacerlo, se pierde la comprensión y la comunicación de su novedad. (Barrera, 2008, p.24)

Estas razones expuestas por investigadores de la comunicación apuntan hacia nuevos enfoques que se analizan en Cuba para un trabajo interdisciplinario comunicativo por variados especialistas de diversas áreas del conocimiento.

En consecuencia, se considera a la enseñanza y el aprendizaje, como procesos de comunicación, que tienen como unidad básica al texto (macro) en su acepción más general, cada uno engloba un sistema de textos que se construyen, analizan, comprenden y aplican a la solución de problemas, téngase en cuenta que en ellos participan docentes y estudiantes, en aras siempre de lograr la comunicación.

Estos estudios han generado definiciones acerca del particular y se retoman dos de ellas.

$\checkmark \quad$ La interdisciplinariedad comunicativa se origina como un intento por ofrecer un resultado favorable a determinados problemas que ni las didácticas particulares de las ciencias, ni la didáctica de la ciencia por separado, ha podido ofrecer una respuesta adecuada. Entre estos problemas se encuentran los bajos resultados en el aprendizaje y la inadecuada comprensión de las ciencias.

$\checkmark$ Se asume la Interdisciplinariedad comunicativa desde una perspectiva que, a partir de una comunicación interdisciplinaria, sistematiza las relaciones de esta naturaleza en el currículo y el desarrollo de éste en la práctica. Tiene como interobjeto la comunicación, y como nodos, las funciones y los componentes de su estructura, particularmente, el signo, el lenguaje, el texto y sus métodos. 
Al mismo tiempo, ella puede concebirse como un proceso circular que comienza en el diagnóstico de un problema: educativo, de aprendizaje o del desarrollo, y transita por los niveles epistemológico, teórico, metodológico y pragmático; presupone el establecimiento de relaciones comunicativas interdisciplinarias. (Barrera, 2010,p. 36)

Pero cuando se analiza la necesidad de la interdisciplinariedad desde el plano teórico y práctico, se requiere ofrecer el cómo hacerlo desde lo metodológico y didáctico, para lo cual se indican los siguientes nodos interdisciplinarios.

1. Los elementos del conocimiento en primer orden.

2. La relación entre los objetivos y las habilidades en las disciplinas

3. Las formas de organización de la docencia.

4. Los métodos productivos en la enseñanza.

5. Los indicadores para evaluar en el proceso educativo a los estudiantes.

Para demostrar a los maestros en formación de la carrera Primaria, se realizan un conjunto de ejercicios y tareas integradoras que permiten corroborar el desarrollo de su pensamiento, y la asimilación de nuevos modos de actuación para diseñar actividades interdisciplinarias en sus contextos rurales cuando realizan su componente laboral investigativo y cuando se evalúan en disciplinas como las didácticas particulares.

Pero para profundizar en cada uno de estos aspectos valorados se particulariza qué se entiende entonces por elementos del conocimiento "la porción de la información que posee un sentido lógico que debe aprender el alumno, caracterizado por su presentación en forma de concepto, ley, hecho, método, proceso, habilidad, principio, etcétera, y cuya amplitud está en dependencia del diagnóstico del proceso de enseñanza-aprendizaje. (Caballero, 2004, p. 8)

A partir de la temática seleccionada en cada uno de los elementos del conocimiento en las disciplinas, se hallan los nodos interdisciplinarios. El nodo interdisciplinario, según criterios de Fiallo es la agrupación del contenido en el que convergen elementos de este correspondientes a distintas disciplinas. (Fiallo, 2003, p.7). En función del alcance del nodo interdisciplinario, este puede ser general (representando el reflejo más profundo y universal de la realidad interdisciplinaria y específico (si se deriva del general y refleja las propiedades más específicas de la realidad interdisciplinaria objeto de estudio).

Los nodos interdisciplinarios se determinan a partir de dos requerimientos básicos, uno de ellos es la precisión de los elementos del conocimiento de las disciplinas con las cuales se va a establecer la interdisciplinariedad, y el otro es el análisis del contenido objeto de estudio en un momento dado, para que en función de ello se utilicen las habilidades, los objetivos, las formas de organización de la docencia, los valores asociados a él que sirven de base para el logro de la interdisciplinariedad.

Sin embargo, existe un enfoque posterior que alude que no es posible hablar de nodos interdisciplinarios, si no se concibe un interobjeto esto es planteado por el Dr. García Ruiz quien lo define como "ente común de cada una de las asignaturas que serán objeto del trabajo interdisciplinario" y qué plantea este especialista, puede estar en el sistema de conocimientos, habilidades, valores, métodos e inclusive según sus propias investigaciones hasta en el modo de actuación de los estudiantes, por ejemplo, ante la resolución de problemas. 
Rosario Mañalich desde la década del 1990, ha incursionado en las humanidades desde el trabajo del Colectivo del Preuniversitario, en busca desde lo metodológico de los puntos de encuentros en los contenidos. Pero el proceso es un todo holístico, por ello, se hace pertinente comprender la necesidad de integrar en el hombre como una unidad, los sentimientos, el pensamiento y la actuación, particularmente, para la formación de la competencia comunicativa de la ciencia con un carácter transversal, lo cual se debe manifestar en el desempeño comunicativo de estudiantes y profesores.

Estas ideas se consideran antecedentes, y a su vez, fundamentos de la Didáctica comunicativa de las ciencias como una teoría didáctica interdisciplinaria, que asume la interdisciplinariedad comunicativa desde una perspectiva que posibilite la comunicación como vía para el logro de relaciones de esta naturaleza en el currículo. Por tanto, en la práctica se tiene como interobjeto a la comunicación y como nodos a las funciones y los componentes de su estructura, particularmente, el signo, el lenguaje, el texto y sus métodos.

Se utilizaron desde los nodos del contenido temáticas afines a otras disciplinas en las clases de Español a partir de la utilización de los diferentes discursos ofrecidos en Práctica Integral de la lengua Española, como exposición, debate, conversación el trabajo con los artículos como tipo de discurso académico. Ejemplo conversar acerca del trabajo político ideológico, temas como la sexualidad, la promoción de salud la vida y obra de Frank País García entre otros temas, ambas temáticas pertenecen a varias asignaturas. (Curso optativo de sexualidad, Tradiciones pedagógicas, Debate y reflexión, Introducción a la especialidad).

Se coordinó por la carrera la utilización de habilidades determinadas por plazos en cada una de las disciplinas, lo que con una adecuada incorporación de las invariantes de cada una de ellas, permitió el mejor logro de los objetivos en los contenidos de las diferentes asignaturas.

La realización por todos los docentes de preguntas escritas que posibiliten evaluar, pero al mismo tiempo ser riguroso con la aplicación de la resolución ortográfica. El mural ortográfico, el cual se actualiza a partir de incorporar palabras claves de cada una de las disciplinas que trabajan diariamente.

Las actividades evaluativas ya fueran clases evaluativas, o evento de la carrera, permitieron tener en cuenta métodos productivos como el trabajo independiente, conversación heurística entre otros, para el desarrollo de los tres niveles de comprensión por todos los profesores: traducción, interpretación y extrapolación incluyendo actividades con situaciones comunicativas. Desde esta óptica se logró una adecuada orientación del estudio independiente que estimulara una buena preparación por el estudiante.

El tratamiento didáctico brindado desde esta perspectiva interdisciplinaria permite solucionar las dificultades con el uso de la lengua materna tanto en el código oral como escrito y revela una atención sistemática por todos los docentes quienes evalúan a partir de los mismos indicadores, lo cual se revierte en un salto cualitativo superior del aprendizaje de los estudiantes. 
Las actividades prácticas potenciaron un carácter dialógico necesario para el tratamiento a las habilidades y el cumplimiento de los objetivos a partir de incorporar métodos productivos y evaluaciones con un mayor rigor científico.

Esta dinámica grupal favoreció el disfrute, el placer del aprendizaje, la alegría, aspectos que convierten a las actividades grupales en el espacio ideal para realizar un aprendizaje realmente efectivo, donde se produce más fácilmente la transformación de los educandos en futuros especialistas.

Se elaboró un Compendio de definiciones principales en la didáctica de la lengua materna, en aras de que todos los docentes en sus disciplinas utilicen las mismas palabras para dar atención a los mismos contenidos. Por ejemplo, cuando se habla de párrafo aludir a texto y sus rasgos esenciales que son la coherencia y cohesión. Otras palabras claves esenciales son el discurso, la semiótica, la semántica, el léxico, la sintaxis entre otras que posibiliten moverse en una misma dirección. Así como las diferencias existentes entre lenguaje, lengua, habla y norma.

La actividad científico estudiantil, así como la realización de pruebas parciales integradoras en el 4 y 5 años de la carrera Primaria, promueven las posibilidades en los estudiantes de prepararse para su futura labor profesional y desde esta óptica, un aporte significativo han sido los trabajos de curso y de diploma que revelan los ejercicios o tareas integradoras desde una perspectiva interdisciplinaria y comunicativa.

Se ejemplifican algunas de estas actividades.

\section{ACTIVIDAD \#1.}

Trabajo de Diploma en el último curso de la carrera, dirigido a los escolares de 5 y 6 grados escuela rural.

Tema: Lectura expresiva del cuento La familia gritería

Objetivo: Leer de forma fluida el texto de modo que les permita a los escolares realizar una comprensión lectora en aras del desarrollo de la lengua materna.

Orientaciones: leer en silencio, oral y formulación de preguntas de los tres niveles.

Forma de organización: clase de consolidación.

Forma de evaluación. Frontal.

Motivación. Narrar el fragmento del cuento y preguntarles. De qué trata el mismo. Técnica de la predicción.

1.1. Del cuento leído responda 
- ¿Cómo es la familia de José '?

- Crees tú correcta la actitud de la familia de José.

- Valora por qué el niño está tan solitario y aislado como una estatua.

- Sabes tú qué es una estatua. En qué asignatura has profundizado sobre este tema tan interesante. ¿Qué otras estatuas están cerca de la escuela?.

- Escribe un texto pequeño a la familia de José, donde le digas como el ruido afecta la vida de la comunidad y las relaciones ambientales. Ten en cuenta, los rasgos caligráficos que debes cumplir y una buena ortografía.

- Crees correcta la indisciplina social en el cuento leído. ¿Qué sucede hoy en tu comunidad con respecto a estas indisciplinas.

Están presentes las relaciones entre Lengua Española, Cívica, Plástica, Historia de Cuba y El mundo en que vivimos.

\section{CONCLUSIONES}

La sistematización de los estudios y enfoques de la interdisciplinariedad comunicativa, desde las Disciplinas Estudios lingüísticos y literarios, ha posibilitado un tratamiento sistemático a la lengua desde una perspectiva integradora en varias asignaturas de los grupos y años.

La aplicación de ejercicios, pruebas parciales y tareas integradoras, posibilita desarrollar modos de actuación en los estudiantes en formación, que los prepara para su trabajo en la escuela primaria, con énfasis para el logro de la integración de los contenidos y objetivos de los grados en la escuela rural.

Asimismo, promueve una atención holística a la lengua materna, lo que favorece una mayor calidad en los estudiantes y una mejor preparación teórico- didáctica para la realización del componente laboral investigativo. 


\section{REFERENCIAS BIBLIOGRÁFICAS}

Abascal, A. (1988). El desarrollo de la lengua materna en la educación cubana. Editorial Pueblo y Educación. La Habana.

Abello, M. (2006). El establecimiento de nexos interdisciplinarios a través del análisis semiótico de textos en diversos estilos funcionales. En Español para todos. Editorial Pueblo y Educación. La Habana.

Addine, F. (1995). Principios para la dirección del proceso pedagógico. La Habana. ISPEJV.

Álvarez, M. (2005). Interdisciplinariedad: una aproximación desde la enseñanzaaprendizaje de las ciencias. Pedagogía a tu alcance, Colección Futuro.

Barrera, J. (2003). Interdisciplinariedad, comunicación y tareas didácticas en la enseñanza de la Física del preuniversitario. [CD-ROM] IV congreso Internacional de Didácticas de las Ciencias. Ciudad de la Habana UNESCO.

Barrera, J. y Cisneros, S. (2002): Una mirada a la necesidad de transformación esencial del currículum de los futuros profesores de Física desde la perspectiva de la comunicación y de una nueva visión de la ciencia. [CDROM] Congreso Internacional "Didáctica de las Ciencias" y VII Taller Internacional sobre la enseñanza de la Física. Instituto Pedagógico Latinoamericano y Caribeño. Cátedra UNESCO en Ciencias de la Educación. La Habana.

. (2010). Hacia una sistematización de la interdisciplinariedad comunicativa desde el trabajo metodológico. Santiago de Cuba.

Caballero, C. (2004). Interdisciplinariedad y currículo en América latina: una estructura didáctica para las ciencias. Universidad de Ciencias Pedagógicas Enrique José Varona. La Habana.

Fernández, A. y otros. (1998). Comunicación educativa. La Habana: Editorial Pueblo y Educación.

Fiallo, J. (1997). Las relaciones interdisciplinarias: una vía para incrementar la calidad de la educación. La Habana: Editorial. Pueblo y Educación.

García, D. (1978). Didáctica del Idioma Español. La Habana: Editorial Pueblo y Educación.

González, R. (1995). Comunicación, personalidad y desarrollo. La Habana: Editorial Pueblo y Educación

Mañalich, R. (2005). Didáctica de las humanidades. La Habana: Editorial Pueblo y Educación. 
Mañalich, R. (1999). Interdisciplinariedad y didáctica: vías para la transformación del desempeño profesional. La Habana: Editorial Pueblo y Educación.

Ojalvo, V. (2004). La comunicación en el aula: su investigación y entrenamiento. CEPES. Universidad de la Habana. Revista Cubana de Educación. Vol. 14. No1.

Ortiz, E. (1995). El estilo comunicativo del maestro. Evento Internacional Pedagogía Palacio de las Convenciones. La Habana.

Rodríguez, M. (2010). El taller metodológico una variante para la introducción del enfoque interdisciplinario comunicativo. Universidad de Ciencias Pedagógicas. Santiago de Cuba.

Roméu, A. (1994). Comunicación y enseñanza de la Lengua materna. Revista Educación \#83. La Habana. 